\title{
La place de la fiction dans le second volume de Bouvard et Pécuchet
}

\section{Stéphanie Dord-Crouslé}

\section{(2) OpenEdition \\ 1 Journals}

Édition électronique

URL : http://journals.openedition.org/aes/579

DOI : 10.4000/aes.579

ISSN : 2258-093X

Éditeur

Laboratoire LISAA

Référence électronique

Stéphanie Dord-Crouslé, "La place de la fiction dans le second volume de Bouvard et Pécuchet ", Arts et Savoirs [En ligne], 1 | 2012, mis en ligne le 15 février 2012, consulté le 01 mai 2019. URL : http:// journals.openedition.org/aes/579; DOI : 10.4000/aes.579

Ce document a été généré automatiquement le 1 mai 2019.

Centre de recherche LISAA (Littératures SAvoirs et Arts) 


\title{
La place de la fiction dans le second volume de Bouvard et Pécuchet
}

\author{
Stéphanie Dord-Crouslé
}

1 Avant d'émettre des hypothèses sur la place que la fiction aurait pu tenir dans le second volume de Bouvard et Pécuchet, il est nécessaire de définir ce qu'on entend par «second volume ». Pour cela, on reviendra sur l'histoire d'un objet matériel et patrimonial complexe dont il faut démêler ce qui en fait, d'une part, un ensemble dont certains éléments peuvent légitimement prétendre à une édition qui se présenterait dans une relative continuité avec celle du "premier volume $»^{1}$, et, d'autre part, une construction historique et intellectuelle à jamais instable et conjecturale, dont les configurations successives se sont trouvées modifiées au fur et à mesure que diverses personnes autorisées (depuis Maupassant jusqu'aux critiques modernes) en ont proposé qui une interprétation et qui une édition.

2 Une fois les limites du second volume identifiées aussi précisément que possible, on pourra comparer les documents existants avec les pages de scénarios laissées par Flaubert; on convoquera aussi ce que l'écrivain a dit dans sa correspondance de la place qu'il allouerait à la fiction, et on se référera à ce que ses proches ont laissé comme indications parcellaires. C'est seulement à la lumière de cette confrontation informée entre l'existant (ou le subsistant?) et les traces scripturales diffuses laissées par le dessein flaubertien que l'on pourra enfin essayer de comprendre dans quelle mesure la fiction aurait innervé le second volume et étayé le surgissement de sa dimension critique ${ }^{2}$.

3 Tâchons tout d'abord de tracer les frontières d'un continent qui - il faut d'emblée l'affirmer et garder le fait toujours présent à l'esprit - est foncièrement mouvant, à savoir le «second volume» de Bouvard et Pécuchet. L'essentiel de ce qui devait servir à sa rédaction se trouve aujourd'hui disséminé dans onze recueils conservés à la Bibliothèque municipale de Rouen depuis 1914³ . En effet, bien avant sa mort en 1931, la nièce de Flaubert et sa légataire universelle, Caroline Commanville puis Franklin Grout, a offert à la ville de Rouen les manuscrits des deux «romans normands" de son oncle, Madame Bovary et Bouvard et Pécuchet. Le registre d'inventaire de la bibliothèque fait mention de ce 
don en date du 13 mai 1914 et propose une brève description de ces documents dont « la consultation [...] est interdite jusqu'au $50^{\mathrm{e}}$ anniversaire de la mort de G. Flaubert, c'est-àdire jusqu'au 9 mai $1930 »$. Les cinq derniers numéros concernent le roman posthume :

- 5). Bouvard et Pécuchet. Manuscrit définitif autographe, en feuilles numérotées de 1 à 215 et de I à XXXIV. Ces 34 feuillets ont été remis au net par une main autre que celle de Flaubert; cette copie se compose de I à XLVI ff et est jointe.

- 6). Brouillon autographe. Paquet de feuilles en désordre sans numérotation suivie, de 0,17 m d'épaisseur (1100 feuilles environ).

- 7). 60 liasses de documents rassemblés par Flaubert en vue de la composition de ce roman (découpures de journaux, extraits de lectures) de diverses mains, notes autographes.

- 8). 1 paquet de fiches rédigées dans le même but.

- 9). Recueil manuscrit non autographe de jugements sur G. Flaubert. $19 \mathrm{ff}$.

On peut présumer que c'est le directeur des bibliothèques de Rouen alors en poste, Henri Labrosse, qui après avoir pris livraison de cet imposant don à Antibes, a procédé pour Bouvard et Pécuchet, comme il l'a fait pour Madame Bovary, au nécessaire et complexe travail de classement des documents en vue de leur conservation patrimoniale; mais on n'en sait pas plus... En tout cas, aujourd'hui ${ }^{4}$, les cinq numéros originels du registre d'entrée se sont métamorphosés en six cotes sous lesquelles sont rangés 22 volumes :

- Ms gg10. Bouvard et Pécuchet. Plans. Manuscrit autographe. 72 feuillets.

- Ms g224 (1) et (2). Bouvard et Pécuchet. Manuscrit autographe (sauf pour les feuillets 251 à 297 inclus). 300 feuillets au total.

- Ms g225 (1) à (9). Bouvard et Pécuchet. Brouillons des chapitres I à X inclus. Manuscrit autographe. 1203 feuillets au total.

- Ms g226 (1) à (8). Recueils de documents divers rassemblés par Flaubert pour la préparation de Bouvard et Pécuchet. 2215 feuillets au total.

- Ms g227. Dictionnaire des idées reçues. Manuscrit en partie autographe. 59 feuillets.

- Ms g228. Dictionnaire des idées reçues. Manuscrit non autographe avec corrections de Flaubert. 26 feuillets.

5 Si l'on peut aisément identifier sous la cote g224 le numéro 5 de 1914, et présumer que le numéro 6 a donné naissance, après reclassement, aux cotes gg10 et g225, il est beaucoup plus difficile de reconnaître les numéros 7, 8 et 9 sous le descriptif des cotes g226, g227 et g2285. Néanmoins, derrière le "paquet de fiches» (numéro 8) se cachent vraisemblablement les folios 2 à 19 du recueil g227, c'est-à-dire l'une des trois versions concurrentes du Dictionnaire des idées reçues; tandis que le descriptif actuel des dossiers permet de retrouver la trace du recueil non autographe de jugements sur Flaubert (numéro 9) dans le huitième volume de la cote g226, folios 208 à $232^{6}$. Quant aux soixante liasses de documents et à leur composition interne originelle, il est impossible de savoir dans quelle mesure elles se retrouvent exactement ou non dans la répartition actuelle des documents en huit volumes (et plus d'une cinquantaine de sous-dossiers) qui constituent aujourd'hui la cote g226.

Les manuscrits de Bouvard et Pécuchet à la Bibliothèque municipale de Rouen

\begin{tabular}{|l|l}
\hline Registre d'inventaire (1914) & Présentation actuelle
\end{tabular}




\begin{tabular}{|c|c|}
\hline $\begin{array}{l}\text { 5). Bouvard et Pécuchet. Manuscrit définitif } \\
\text { autographe, en feuilles numérotées de } 1 \text { à } 215 \text { et } \\
\text { de I à XXXIV. Ces } 34 \text { feuillets ont été remis au } \\
\text { net par une main autre que celle de Flaubert; } \\
\text { cette copie se compose de I à XLVI ff et est } \\
\text { jointe. }\end{array}$ & $\begin{array}{l}\text { Ms g224 (1) et (2). Bouvard et Pécuchet. } \\
\text { Manuscrit autographe (sauf pour les feuillets } \\
251 \text { à } 297 \text { inclus). } 300 \text { feuillets au total. }\end{array}$ \\
\hline $\begin{array}{l}\text { 6). - Brouillon autographe. Paquet de feuilles en } \\
\text { désordre sans numérotation suivie, de } 0,17 \mathrm{~m} \\
\text { d'épaisseur ( } 1100 \text { feuilles environ). }\end{array}$ & $\begin{array}{l}\text { Ms gg10. Bouvard et Pécuchet. Plans. } \\
\text { Manuscrit autographe. } 72 \text { feuillets. } \\
\text { Ms g225 (1) à (9). Bouvard et Pécuchet. } \\
\text { Brouillons des chapitres I à X inclus. } \\
\text { Manuscrit autographe. } \\
1203 \text { feuillets au total } 7 \text {. }\end{array}$ \\
\hline $\begin{array}{l}\text { 7). - } 60 \text { liasses de documents rassemblés par } \\
\text { Flaubert en vue de la composition de ce roman } \\
\text { (découpures de journaux, extraits de lectures) } \\
\text { de diverses mains, notes autographes. }\end{array}$ & $\begin{array}{l}\text { Ms g226 (1) à (8). Recueils de documents } \\
\text { divers rassemblés par Flaubert pour la } \\
\text { préparation de Bouvard et Pécuchet. } 2215 \\
\text { feuillets au total [y compris ceux qui } \\
\text { correspondent au 9)]. } \\
\text { Ms g227. Dictionnaire des idées reçues. } \\
\text { Manuscrit en partie autographe. } 59 \\
\text { feuillets [y compris ceux qui } \\
\text { correspondent au 8)]. } \\
\text { Ms g228. Dictionnaire des idées reçues. } \\
\text { Manuscrit non autographe avec } \\
\text { corrections de Flaubert. } 26 \text { feuillets. }\end{array}$ \\
\hline $\begin{array}{l}\text { 8). - } 1 \text { paquet de fiches rédigées dans le même } \\
\text { but. }\end{array}$ & $\begin{array}{l}\text { Ms g227 fo à } 19 \text {, soit l'une des trois } \\
\text { versions du Dictionnaire des idées reçues. }\end{array}$ \\
\hline $\begin{array}{l}\text { 9). Recueil manuscrit non autographe de } \\
\text { jugements sur G. Flaubert. } 19 \mathrm{ff} \text {. }\end{array}$ & $\begin{array}{l}\text { Ms g226 (8) for à 232, ensemble de } \\
\text { jugements sur Flaubert. }\end{array}$ \\
\hline
\end{tabular}

$6 \mathrm{Au}$ premier abord, tous les manuscrits qui auraient pu être utilisés par l'écrivain pour composer le second volume de son œuvre semblent donc être aujourd'hui regroupés dans les 2215 feuillets de la cote g226, auxquels il faut ajouter les deux volumes du Dictionnaire des idées reçues et le recueil des "Plans ${ }^{8}$. Cependant, les choses ne sont pas aussi simples : il y a ici à la fois excès et manques. En effet, sous la cote g226 sont conservés des documents qui n'ont vraisemblablement rien à voir avec le dernier roman de Flaubert : la nièce Caroline en avait-elle conscience au moment où elle les a donnés à la ville de Rouen? Il est probable que non; mais quelle que soit la raison qui l'a amenée à les réunir, il faut - autant que faire se peut - les extraire du corpus pour cerner au mieux les contours fuyants du second volume?.

7 Ainsi, l'ensemble considérable constitué par les Mémoires de Madame Ludovica (volume 4, $\mathrm{f}$ - 233 à $272 v^{\circ}$ ) a été utilisé comme documentation pour Madame Bovary et n'avait guère de raison de faire retour dans Bouvard et Pécuchet. L'épais dossier de notes de lecture prises 
sur l'Histoire des peintres de toutes les écoles, par Charles Blanc (volume 5, fo 334 à 355), n'a pas non plus sa place dans les documents préparatoires pour le dernier roman ${ }^{10}$, au moins depuis que Flaubert a renoncé aux escapades artistiques parisiennes qu'il avait initialement prévues pour ses deux personnages. Se trouve aussi dans les dossiers de Rouen un important ensemble de pièces de théâtre partiellement rédigées et de scénarios (volume $8, f^{\circ} 42$ à 207) écrits pour la plupart de mains inconnues et dont Yvan Leclerc a récemment proposé un descriptif et une synthèse ${ }^{11}$. Pour montrer à quel point l'entreprise est complexe, il suffit de rappeler que c'est aussi dans cet ensemble théâtral ô combien composite - que l'on découvre, d'une part, le plan originel d'Un cœur simple ( folio 195) et, d'autre part, un résumé-sommaire de L'Éducation sentimentale (folio 206). C'est d'ailleurs sûrement pour préparer ce seul roman que Flaubert s'est documenté sur les arts décoratifs (volume 1, fo 137 à 165) et a rassemblé (vraisemblablement avec l'aide de son ami Jules Duplan) un nombre conséquent de coupures de presse et de professions de foi relatives aux événements de 1848 (volume 2, fo 19 à 166), ainsi que des notes de lecture (volume 4, fo 133 à 204) qui ne semblent pas avoir été réutilisées pour Bouvard et Pécuchet, à la différence d'autres ensembles. Dernier dossier d'importance, les papiers de Duplan. En effet, le cinquième volume contient presque trois cents pages écrites de la main de cet ami de Flaubert mort en 1870 . Or, si certaines pages, comme le célèbre "Album de la marquise » présentent des liens évidents avec l'entreprise du second volume, elles ne font que la préfigurer ${ }^{12}$ et ne sauraient être insérées telles quelles. La meilleure preuve en est que les quelques citations retenues par Flaubert se trouvent marquées en marge et presque toutes recopiées sur des pages préparées pour le second volume.

8 Sous la cote g226, à côté des documents qui ne concernent donc sans doute pas le roman posthume, il y a aussi des ensembles de feuillets dont l'appartenance typologique même les exclut du corpus des pages mobilisables pour la composition du second volume. Il s'agit tout d'abord de documents bruts comme des journaux complets dont on ne sait pour quelle raison ils se trouvent dans les dossiers, de feuillets de recherches bibliographiques qui ont orienté et alimenté les lectures de Flaubert en amont ou en parallèle à la rédaction, mais aussi de pages préparatoires à destination du premier volume du roman (surtout des pages de "notes de notes ", mais aussi quelques brouillons ) voire d'autres œuvres (des brouillons et un croquis concernent L'Éducation sentimentale).

9 Le cas des notes de lecture est plus complexe et nécessite un examen spécifique. Elles devraient logiquement être écartées du corpus. En effet, le processus rédactionnel propre à Bouvard et Pécuchet amenait l'écrivain à se servir de ses notes en deux temps : d'une part, en vue du premier volume de son roman, il reportait sur des pages de «notes de notes" les éléments textuels qu'il avait repérés; et d'autre part, il recopiait (ou faisait recopier par son ami Laporte) d'autres fragments textuels sur des pages particulières à destination du second volume $\mathrm{e}^{13}$. Si Flaubert avait mené à son terme la rédaction de son roman, si les deux types de sélection et de transfert avaient été opérés partout, les notes de lecture auraient livré leur « substantifique moelle » et ces pages pourraient être rejetées en bloc à l'extérieur du premier cercle. Mais deux événements inattendus sont venus contrarier le bel ordonnancement et la "mécanique compliquée $»^{14}$ du travail flaubertien. Le premier est la brouille survenue entre l'écrivain et son ami Edmond Laporte en septembre 1879 - qui a privé le romancier d'un auxiliaire efficace et ô combien nécessaire à ce moment précis, et a considérablement ralenti son travail ; le second est la propre mort de Flaubert le 8 mai 1880 qui a brutalement et définitivement immobilisé le chantier 
documentaire alors que des pages de notes de lecture n'avaient pas été complètement traitées en vue du second volume.

En toute rigueur, il faudrait donc opérer un tri entre les différents dossiers de notes de lecture, exclure les plus anciens, ceux dont on peut penser qu'ils sont effectivement passés au double crible (comme la médecine, l'agriculture ou la politique), et conserver les plus récents, ceux dont certains éléments matériels prouvent qu'ils ont échappé peu ou prou au traitement à destination du second volume. Mais ce classement est fort malaisé à établir, d'autant que des pages de copie destinées au second volume peuvent avoir disparu, et que des signes de sélection pour le second volume, présents sur certaines notes de lecture, ne trouvent de correspondant dans aucune page préparée - sans qu'on puisse décider si c'est parce que Flaubert a finalement renoncé à son choix initial ou parce que la page a été perdue. En outre, selon un scénario, des «notes des auteurs précédemment lus » auraient dû prendre place dans le second volume, bien qu'on ne dispose d'aucune indication précise pour identifier le type de notes visées par Flaubert. Ces incertitudes cumulées poussent à réintégrer dans le corpus la totalité des notes de lecture - une fois précisé que dans les nombreux cas de concurrence qui se présenteront, c'est le fragment textuel recopié sur une page préparée pour le second volume qui primera.

11 S'il y a donc des dossiers en excédent parmi ceux conservés à Rouen, on repère aussi des lacunes. La plus anciennement connue est due à la brouille du romancier avec Edmond Laporte. En effet, ce serviable ami dont Flaubert utilisait la belle main pour mettre au propre les citations destinées à la « copie » (c'est-à-dire au second volume du roman), a conservé le dossier sur lequel il travaillait lorsqu'ils se sont fâchés, soit une "cinquantaine» de citations "port[ant] de la main de Flaubert des indications marginales faites en vue de leur classement $»^{15}$. La trace de ce dossier est aujourd'hui apparemment perdue ${ }^{16}$. Il ne faut pas non plus oublier les ensembles de notes de lecture que la nièce Caroline - pour une raison qui échappe - a choisi de ne pas joindre à son legs de 1914 à la ville de Rouen. Ces différents dossiers sont donc passés en vente après sa mort en 1931. Les catalogues des deux ventes après-décès organisées cette même année gardent la trace de ses ensembles disparus dont certains ont refait surface depuis (comme celui de la Littérature esthétique ${ }^{17}$ ) mais qui demeurent, pour un trop grand nombre d'entre eux, introuvables (Histoire, Géologie, Astronomie, Archéologie celtique...).

$12 \mathrm{Au}$ terme de ce parcours, en l'état actuel des fonds connus, font donc partie des documents mobilisables dans le cadre du chantier du second volume :

- au premier chef, la catégorie des pages dont on peut penser qu'elles sont « préparées pour le second volume » (autour de 450),

- les pages récapitulatives, dont sont issues certaines pages préparées pour le second volume (une petite cinquantaine),

- les notes de lecture, ou plutôt un certain nombre de fragments textuels appartenant à ces notes qui sont désignés pour le second volume par une mention de type « copie », « copiez » ou «à copier », une catégorie de classement (« Beautés » ou «Style»), une croix ou un trait dans la marge,

- la somme ${ }^{18}$ des trois manuscrits concurrents du Dictionnaire des idées reçues,

- et les scénarios qui proposent une organisation partielle ou d'ensemble de ces différents matériaux $^{19}$.

13 Maintenant que l'on discerne un peu moins vaguement les contours à jamais diffus du second volume, on va tenter d'entrer plus avant dans son organisation car ce point est 
central pour qui veut comprendre comment fiction et encyclopédie auraient pu y être agencées. La première à s'être affrontée à cette question est sûrement Caroline ellemême. Légataire universelle et exécutrice testamentaire, c'est à elle qu'est revenue la tâche, après le décès brutal de son oncle, de veiller à la diffusion, au respect et - dans une certaine mesure - à la rentabilité financière de son œuvre... Son premier soin a été d'honorer l'accord projeté ${ }^{20}$ par Flaubert avec Juliette Adam pour la parution de Bouvard et Pécuchet. Rapidement, elle a mis au propre une copie des dix chapitres rédigés - et le roman a paru en six livraisons dans La Nouvelle Revue, du 15 décembre 1880 au $1^{\mathrm{er}}$ mars 1881, puis, la même année, en volume chez Lemerre. Mais Caroline n'a pas oublié que son oncle avait prévu un autre tome: le traité signé avec La Nouvelle Revue précise que «la publication ultérieure de documents laissés par Gustave Flaubert et qui devaient former un volume de notes, présentant la conclusion et la justification philosophique dudit roman ne donnera à Mme Adam aucun droit de reproduire ces documents qui ne constituent pas une œuvre laissée par l'auteur de Bouvard et Pécuchet ${ }^{21}$.

En parallèle avec la copie au net des chapitres rédigés (ou peu après ?), Caroline a mis en ordre les dossiers de son oncle afin de préparer la publication de ce qu'elle nomme le "volume de notes ", c'est-à-dire le second volume. C'est vraisemblablement de cette époque que datent les quatre pages d'inventaire ${ }^{22}$ serrées dans le huitième volume de la cote $\mathrm{g} 226\left(\mathrm{f}^{\circ} 38 \mathrm{à} 39 \mathrm{v}^{\circ}\right)$. Les indications qu'on y trouve sont tout à fait concordantes avec l'écriture et le type des annotations que Caroline a portées sur les différents manuscrits des œuvres de son oncle ${ }^{23}$. L'intérêt de cet inventaire réside en ce qu'il montre ce qui devait entrer, selon Caroline, dans la composition du second volume, en fonction, d'une part, de l'idée qu'elle se faisait du projet de son oncle (projet dont il avait dû abondamment lui parler, mais sans lui en communiquer forcément les infléchissements successifs) et, d'autre part, de l'organisation matérielle des papiers de l'écrivain lorsqu'elle les a découverts. La nièce isole quatre grands ensembles ${ }^{24}$. Le premier est constitué de trois dossiers attendus («Bizarreries - Nomenclatures", "Beautés» et " Histoire et idées scientifiques ») auquel est joint plus curieusement le recueil intitulé « République de $1848 »^{25}$. Le verso du premier feuillet de l'inventaire est occupé par un nouveau rapprochement sans surprise, celui des dossiers "Grands hommes", «Esthétique et critique » et «Styles ». Quant au second feuillet, il agrège des dossiers plus divers mais complétant effectivement (à l'exception de «Journaux $»^{26}$ ) la liste des regroupements de pages destinées au second volume : le " Dictionnaire des Idées reçues ", «Morale», «Religion, Mysticisme, Prophéties», «Morale, Socialisme, Politique», " Amour », « Philosophie », « Exaltation du bas », «Imbéciles » et « Esprit des journaux ».

Le suivant à s'être penché sur ce chantier est Guy de Maupassant. En effet, Caroline a demandé à celui que son oncle appelait son « disciple » de mettre en ordre les documents existants afin de publier le "volume de notes" mentionné dans le traité signé avec Juliette Adam. Elle remet donc à Maupassant, avant la fin de l'année $1880^{27}$, les dossiers qu'elle estime être concernés par l'entreprise (à moins que le jeune homme ne soit venu faire lui-même son choix à Croisset?). En tous cas, les manuscrits se trouvent dans l'appartement de Maupassant au moins jusqu'en août $1881^{28}$. Entre ces deux dates, Maupassant " pass[e] trois mois à compulser et à tenter de disposer les notes [du] pauvre mort, pour en tirer le livre qu'il voulait faire » - avant de renoncer : « je crois maintenant cette besogne inexécutable ». Dans la lettre qu'il envoie à Caroline le 30 juillet 1881, le disciple expose les diverses raisons qui l'amènent à abandonner l'entreprise. En 1884, il sera néanmoins le premier à lever un coin du voile sous lequel reposent les prémices du 
second volume, lorsqu'il publiera de larges extraits des documents "compulsés » dans son étude intitulée "Gustave Flaubert ${ }^{29}$. Outre les nombreuses citations qu'il comporte, cet article présente l'intérêt de proposer un classement ${ }^{30}$ pour cette «montagne de notes demeurées trop éparses, trop mêlées, pour être jamais publiées en entier ». Si Maupassant insiste sur le fait que Flaubert « devait revoir cette classification première, la modifier, supprimer au moins la moitié de cet amas de documents ", il n'en affirme pas moins que cet « ordre dans lequel [Flaubert] a laissé ces notes » constitue le plan (ou du moins le seul plan concevable en l'état) du second volume.

Si des mises en forme typographiques complexes rendent parfois scabreuse la compréhension des arborescences construites par Maupassant, la comparaison de son plan avec l'inventaire de Caroline est cependant instructive. Si la nièce s'en tient scrupuleusement aux titres qu'elle trouve inscrits sur les pochettes, Maupassant tente quelques regroupements plus audacieux, comme «Styles des grands écrivains, des journalistes, des poètes ». La nièce convoque des dossiers (comme «Journaux» et "République de 1848 ») que le disciple n'a pas retenus, à juste titre d'ailleurs; en revanche, celui-ci indique des catégories que celle-là n'a pas mentionnées explicitement, telles «Charabia officiel », « Palinodies » ou « Beaux-arts ». Et si tous deux incluent dans leur liste le Dictionnaire des idées reçues, Maupassant est le seul à intégrer le « catalogue des opinions chic", tandis que Caroline fait seule état de l'existence de l'«Album de la marquise ». En dépit de quelques regroupements spécifiques et d'un niveau de précision variable dans la description des sous-dossiers, les plans proposés par Caroline et Maupassant concordent donc dans leurs grandes lignes et trouvent leur pendant dans l'inventaire des recueils documentaires tels qu'ils sont conservés aujourd'hui à la bibliothèque municipale de Rouen ${ }^{31}$.

17 Mais peut-on confondre ces listes plus ou moins ordonnées en fonction de ce qui existait matériellement au moment de la mort de Flaubert, avec le plan qu'aurait suivi l'écrivain pour écrire le second volume de son roman? La réponse est évidemment négative et Maupassant l'affirme clairement dès 1881 dans sa lettre déjà citée à Caroline. C'est même la raison principale qui l'a fait renoncer à l'entreprise éditoriale confiée par l'exécutrice testamentaire de Flaubert :

Ces notes, dans son projet, devaient être reliées, soudées ensemble, par des morceaux de récit qui remettaient en scène les deux commis, et par des morceaux de dialogues, formant les commentaires de leurs lectures et de leurs copies. Ces parties, je ne puis me permettre de les faire, et, sans elles, le livre est illisible : il ne forme plus qu'une agglomération, qu'un amas de citations sans ordre, dont le sens même échappera très souvent au lecteur. ${ }^{32}$

Flaubert prévoyait même d'insérer de la fiction dans son second volume par un autre biais, cette fois déconnecté de l'histoire de Bouvard et Pécuchet, à savoir en incluant des textes complets qui auraient largement dépassé l'extension habituellement réduite des citations du Sottisier. Ce dessein est indiqué tant par Caroline que par Maupassant. Celuici termine son étude sur «Bouvard et Pécuchet» par la publication in extenso d'une « nouvelle » inédite de plusieurs pages intitulée Une nuit de don Juan:

Gustave Flaubert comptait donc former un volume entier de ces documents justificatifs. Pour rendre moins lourd et fastidieux ce recueil de sottises, il y aurait intercalé deux ou trois contes, d'un idéalisme poétique, copiés aussi par Bouvard et Pécuchet.

On a trouvé dans ses papiers le plan d'une de ces nouvelles, qui aurait été intitulée : Une Nuit de Don Juan.

Ce plan, indiqué en phrases courtes, souvent même par des mots sans suite, révèle 
mieux que toute dissertation sa manière de concevoir et de préparer son travail. À

ce point de vue, il peut être intéressant. ${ }^{33}$ œuvre. Si l'on se réfère à la première mention explicite de Bouvard et Pécuchet dans la Correspondance, la bipartition du roman (sa composition en deux volumes) n'apparaît même pas comme telle dans cette rapide évocation :

C'est l'histoire de ces deux bonshommes qui copient, une espèce d'encyclopédie critique en farce. Vous devez en avoir une idée ? Pour cela, il va me falloir étudier beaucoup de choses que j'ignore: la chimie, la médecine, l'agriculture. Je suis maintenant dans la médecine. - Mais il faut être fou et triplement frénétique pour entreprendre un pareil bouquin! Tant pis, à la grâce de Dieu !36

L'intrigue se présente comme un tout - sans qu'on puisse dissocier le parcours encyclopédique en acte des deux bonshommes (parcours premier mais ici complètement passé sous silence) de sa duplication livresque ultérieure dans le processus de la copie. Flaubert présente d'un seul tenant les manières successives dont les disciplines scientifiques et les ouvrages qui les exposent vont surgir (expérimentation puis copie), unissant dans une même phrase l'intrigue fictionnelle réduite à sa portion congrue (seulement le début et la fin, c'est-à-dire les deux moments où Bouvard et Pécuchet ne font effectivement que copier) et l'effet de lecture synthétique qu'il faudra avoir produit dans l'esprit du lecteur lorsque celui-ci arrivera au terme de la fiction complète : toute l'intrigue se trouve ainsi ramassée dans une expression qui en agrège les deux facettes.

Cette prééminence accordée à la copie sur les aventures des deux personnages dans les différentes contrées du savoir se lit aussi dans la manière dont Flaubert désigne parfois son roman dans sa correspondance. En effet, dans la première phase de conception, l'écrivain appelle à plusieurs reprises son œuvre en devenir: Les deux copistes ${ }^{37}$. Néanmoins, si le titre n'est pas anodin, la cause de son emploi doit aussi être cherchée dans la chronologie de la rédaction du roman - et donc dans la succession des différents 
éléments de l'intrigue: dans ce que Flaubert a d'abord nommé l'introduction ou le prologue du roman (avant qu'il n'en devienne le premier chapitre), Bouvard et Pécuchet noircissent des registres chacun de leur côté sur le banc d'une maison de commerce. Ils remplissent donc alors effectivement un rôle de copistes - comme ils seront plus tard archéologues, acteurs ou philosophes. D'ailleurs, cette formulation n'apparaît jamais dans les scénarios où le roman est d'abord intitulé : «Histoire de deux Cloportes. Les deux commis » (carnet 19, $\mathrm{f}^{\circ} 41$ ), puis, après quelques hésitations onomastiques, "Bouvard et Pécuchet ", dès le premier scénario de Rouen ${ }^{38}$ ( $f^{\circ} 2$ du recueil gg10).

L'exigence de fiction et le nécessaire recours à son pouvoir se sont fortement imposés à Flaubert. Que le ressort fictionnel se détende ou soit seulement pris en défaut, et l'œuvre vacille voire se défait sans tarder: " il faut un semblant d'action, une espèce d'histoire continue pour que la chose n'ait pas l'air d'une dissertation philosophique $"^{39}$, ce qui impose, en complément et comme soutènement à la dimension encyclopédique d'ensemble, «des dialogues, de petites scènes et des personnages secondaires! $»^{40}$. La donnée fictionnelle est donc un souci constant pour l'écrivain qui se réjouit lorsque «[s]es bonshommes se dessinent dans [s]on esprit » ${ }^{41}$ ou qu'il les « entrevoi[t enfin] d'une façon plus vivante et moins artificielle $»^{42}$. Cette préoccupation a des répercussions sur l'économie générale de l'œuvre que Flaubert conçoit rapidement comme un roman de grande ampleur, lorsque Tourgueneff lui conseille au contraire de faire court : " plus j'y rêve, plus c'est un sujet à traiter presto, à la Swift, à la Voltaire. Vous savez que ça a toujours été mon opinion. Votre scénario raconté m'a semblé charmant et drôle. - Si vous

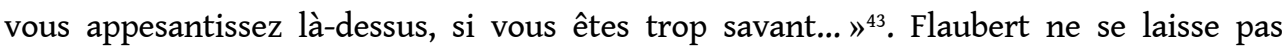
intimider par la sourde menace que font planer les points de suspension dissuasifs employés par son ami ; il lui rétorque aussitôt : si ce sujet « est traité brièvement, d'une façon concise et légère, ce sera une fantaisie plus ou [moins] spirituelle, mais sans portée et sans vraisemblance, tandis qu'en détaillant et développant, j'aurai l'air de croire à mon histoire, et on peut faire une chose sérieuse et même effrayante $»^{44}$. Il n'est donc pas question d'abandonner en chemin l'un des deux termes: la fiction doit permettre à l'encyclopédie de se déployer tandis que l'encyclopédie doit fonctionner comme le ressort essentiel de la fiction.

Pourtant, tout un pan de la Correspondance peut laisser supposer que le projet de Flaubert n'a pas atteint son objectif et que ce rêve d'heureuse fusion entre les deux dimensions est un échec: en 1875, l'écrivain affirme s'être "lancé dans une entreprise absurde " ${ }^{45}$, entreprise dont il doute toujours de la validité quatre ans plus tard : « Quel livre ! [...] Et la conception en est peut-être vicieuse ? Il est peut-être impossible de faire un roman avec cette idée-là ? C'est trop voulu. Il n'y a jamais eu deux bonshommes pareils ? $»^{46}$. À l'en croire, la fiction se serait fait la trop humble servante de l'encyclopédie, jusqu'à disparaître quasiment. Flaubert ne présente-t-il pas son œuvre comme "une espèce d'encyclopédie de la Bêtise moderne $»^{47}$ ? N'a-t-il pas peur que «ce ne soit embêtant à crever $»^{48}$ ? Et si son «but (secret)» est d'« ahurir tellement le lecteur qu'il en devienne fou », ce but, explique-t-il, «ne sera pas atteint, par la raison que le lecteur ne [le] lira pas. Il se sera endormi dès le commencement $~^{49}$. L'encyclopédie aurait finalement dévoré la fiction, rendant toute lecture romanesque impossible. Flaubert prévient ainsi Edma Roger des Genettes que, dans son roman, « la volupté [...] tient autant de place que dans un livre de mathématiques! et pas de drame, pas d'intrigue ! pas de milieu intéressant ! $\aleph^{50}$; quant à Gertrude Tennant, il lui affirme que Bouvard et Pécuchet est « une revue de toutes 
les idées modernes. Les femmes y tiennent peu de place et l'amour aucune. [...] Ceux qui lisent un livre pour savoir si la baronne épousera le vicomte seront dupés $»^{51}$.

Mais ce constat n'est pas un aveu d'échec; c'est une manière détournée pour Flaubert de préparer ses lecteurs à l'établissement d'un régime fictionnel inhabituel. La fiction ne se retire pas du roman ; au contraire, elle est véritablement au cœur de l'ultime entreprise flaubertienne, et ce cœur étend ses ramifications aussi bien du côté du premier que du second volume. L'analyse des scénarios de la fin du roman va permettre de le montrer.

Les plus anciens appartiennent au carnet de travail numéroté 19 et datent vraisemblablement de 1862, période à laquelle, au terme de la rédaction de Salammbô, Flaubert hésite entre deux projets : celui qu'il choisira finalement de traiter, L'Éducation sentimentale, et celui qu'il laissera de côté pendant presque dix ans, le futur Bouvard et Pécuchet. À cette époque, le scénario du roman connait une élaboration en deux volets. Sur le folio 41, d'abord, les éléments fictionnels sont distribués en trois ensembles numérotés par des chiffres romains. Le premier est consacré à la rencontre des deux personnages à Paris et à leurs rêves d'évasion hors de la ville ; il se clôt sur leur départ et leur premier réveil en un lieu non précisé, mais sûrement éloigné de la capitale. La seconde partie évoque leurs occupations, d'abord restreintes au seul cercle étroit de ce qui est praticable à la campagne (jardinage, chasse, pêche, agriculture), puis le dépassant dans un premier accès d'encyclopédisme débridé (littérature, histoire, socialisme, métaphysique, religion, sciences, éducation...). Mais cet élan euphorique se trouve aussitôt contrebalancé par la mention de l'incommensurable ennui qui finit par saisir les personnages : ils épient les diligences et l'un d'entre eux effectue même à Paris un voyage teinté de nostalgie. La troisième partie, quant à elle, qui devrait logiquement offrir une échappatoire et permettre de dépasser l'aporie, se trouve d'abord à peine ébauchée par deux notations sibyllines: «III. bonne idée. lyrisme de la faim fin ». Elle connaît ensuite une reformulation et un développement notable sur le feuillet qui, quand on feuillette le carnet 19 , fait face à celui qui vient d'être commenté, soit le folio $40 \mathrm{v}^{\circ}$ :

Bouvard et Pécuchet, folio $40 \mathrm{v}^{\circ}$

ils copient tout ce qui leur tombe sous la main (ne pouvant faute

etc.

d'argent avoir des livres) cornets de papier ete. à tabac. on peut insérer en faits

là tout ce que l'on veut comme contrastes, pastiches de style.

mettre des morceaux vrais $\&$ des morceaux

\author{
typiques \\ des extraits de critique idiots \\ dans tous les genres.
}

folio 41 , la liste suivante :

dans les copies

tableaux parallèles

antithétiques.

crimes des rois 
\& des peuples

bienfaits

sont qqfois

embarrassés. - Cas de conscience.

29

Le développement encyclopédique des aventures des deux cloportes au-delà des simples activités campagnardes semble ainsi avoir été conçu simultanément avec cette seconde élaboration du contenu de la copie: le tissage intime des dimensions fictionnelle et encyclopédique prend sa source ici et montre qu'il a vocation à fonctionner aussi bien pour le premier que pour le second volume. En effet, si Bouvard et Pécuchet s'ennuient de l'examen successif des disciplines, ils sont «embarrassés» et ont des «cas de conscience » dans leur activité de copie ${ }^{52}$.

Ensuite, dans le recueil des scénarios (ms gg10) conservés à la bibliothèque municipale de Rouen, la fin du roman connaît quatre élaborations successives ${ }^{53}$. La première ( $\left.f^{\circ} 5\right)$ fait vraisemblablement partie de ces 《quatre pages de scénario $»^{54}$ dont Flaubert parle à sa nièce Caroline au mois d'août 1872. La matière de l'ensemble du roman y est encore organisée en trois parties, le dernier feuillet seul traitant de la fin. On y retrouve tous les éléments présents dans le carnet 19 , augmentés d'une indication sur la manière dont les personnages résolvent leurs « grands cas de conscience »:

Bouvard et Pécuchet, carnet 19

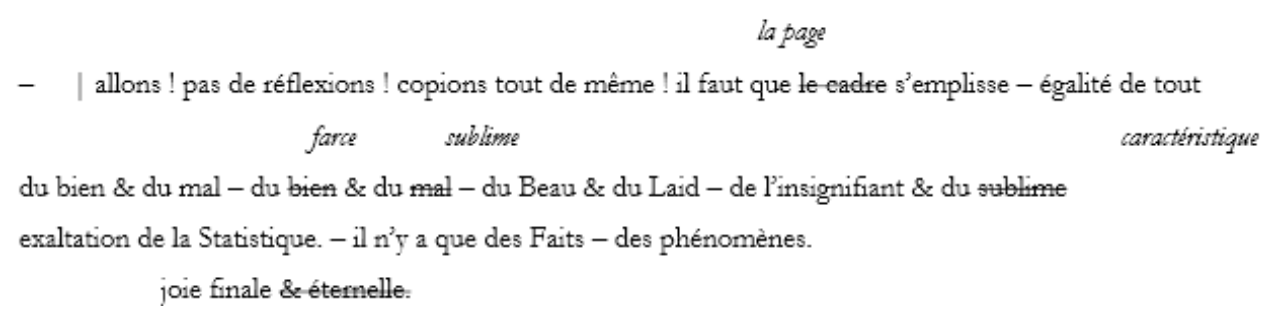

31 L'occurrence suivante, développée sur le folio 19, porte la trace d'un remaniement en profondeur de l'architecture du roman : le chiffre « III » accompagné de la notation « Leur copie » est raturé et remplacé par le chiffre « $\mathrm{X}$ ». La structure ternaire de l'œuvre est abandonnée au profit d'un agencement de la matière romanesque en chapitres. En outre, ce feuillet présente plusieurs avancées scénariques notables. D'abord, pour expliquer l'origine des papiers que Bouvard et Pécuchet commencent par copier au hasard, Flaubert imagine qu' i il y a aux environs une fabrique de papier, en faillite. Ils achètent au poids quantité de vieux papiers - salis \& déchirés ». Ensuite, le romancier ajoute à la liste des classements possibles pour le contenu de la copie la question des différents styles, ce qui permet de convoquer et de décliner nombre de champs encyclopédiques successivement rencontrés par les deux personnages : « morceaux de style. médical, agricole - politique, officiel - critique littéraire». Mais surtout Flaubert a l'idée d'ajouter un tour supplémentaire à la spirale sur laquelle il construit son intrigue : 


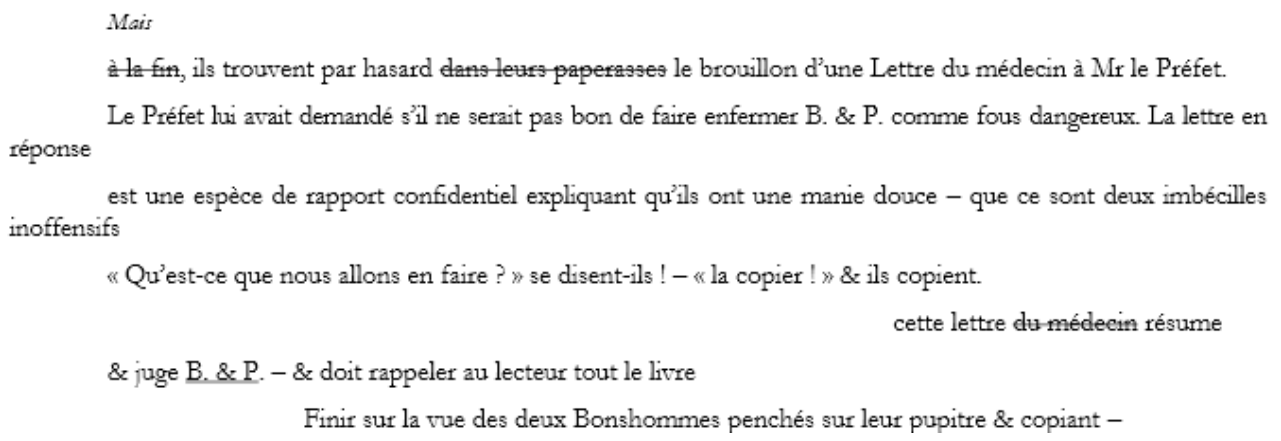

Ce procédé de la lettre trouvée " par hasard », en ce qu'elle « doit rappeler au lecteur tout le livre", induit un nouveau tour pour la roue encyclopédique mais vient aussi unir toujours plus intimement la fiction et l'exposition des savoirs.

Cette dynamique se poursuit dans le scénario suivant qui se trouve sur le folio 32. Intitulé «Leur copie » et numéroté «XI », il reprend tous les éléments du scénario précédent et en invente de nouveaux. En particulier, il propose de jeter un :

Coup d'œil sur ce qui s'est passé dans

le village, depuis qu'ils sont à copier.

Marescot a quitté Chavignolles, a fait

des spéculations \& est notaire à Paris.

Mélie entrée chez Beljambe, comme

servante l'a épousé - lui mort, elle

se marie à Gorgu \& trône à l'auberge.

Il révèle aussi une nouvelle et importante réorganisation de la matière puisqu'un chiffre «XII » apparaît en face de la découverte du brouillon de la lettre du médecin : l'ultime chapitre sera donc entièrement consacré à cette copie très particulière, et c'est sur elle qu'il est prévu que le roman s'achève. Cette répartition de la matière est confirmée dans l'occurrence suivante ( $\left.f^{\circ} 67\right)$ : «XI = Leur copie » et « XII = conclusion ». Dans le dernier chapitre, la lettre qu'adresse le médecin au préfet, « en résumant toutes leurs actions \& pensées, [...] doit, pr le lecteur, être la critique du roman » - et cette critique - ou plutôt la critique en creux que le lecteur habile en aurait fait, n'aurait pas été en demi-teintes ${ }^{55} . .$. Le dispositif esquissé, toutes proportions gardées, rappelle curieusement la toute fin de Madame Bovary, entièrement occupée par les succès et le triomphe ultime de Homais, le médecin Vaucorbeil prenant ici la place du pharmacien. Simultanément, l'avant-dernier chapitre se voit rattacher une dizaine de catégories de classement et/ou types de documents destinés à apparaître dans le «Monument » des deux bonshommes et qui servent de base - depuis lors - aux différentes expériences de reconstitution conjecturale du second volume : 


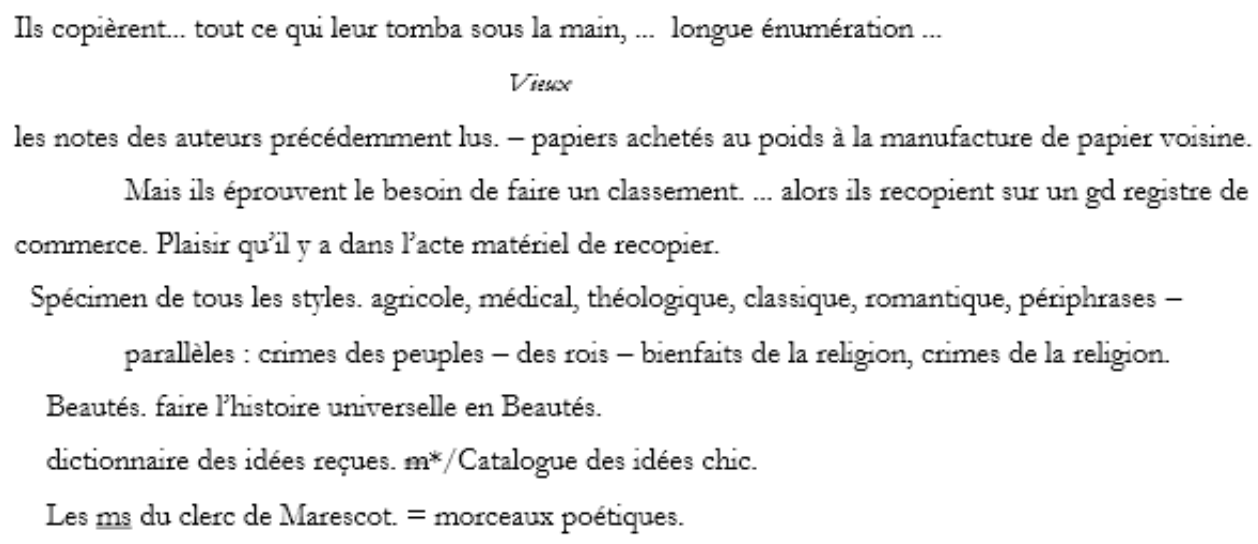
Annotations au bas des Copies.

Reste cependant un fragment scénarique (sur le fo 68) qu'il est difficile de faire fonctionner avec les autres :

Bouvard et Pécuchet, $f 68$

avant la Copie, après l'introduction, mettre en italique, ou en note. « un hazard a permis de " on a retrouvé par hazard leur copie, l'Éditeur la donne afin de grossir le présent ouvrage »

La seule manière de comprendre cette note est de la rattacher au moment révolu de la conception scénarique qui a précédé l'invention de la lettre au préfet. Il aurait alors été logique de proposer un épisode narratif plus ou moins développé («l'introduction») expliquant en quoi consiste la copie des deux bonshommes et le bonheur final qu'ils y trouvent - et de repousser les extraits eux-mêmes dans une sorte d'appendice au roman introduit par un avertissement de l'éditeur. Dans ce cas, la retranscription de la copie aurait vraisemblablement été exempte de toute notation fictionnelle appartenant à l'univers des deux bonshommes : on aurait lu in extenso le registre de commerce sur lequel Bouvard et Pécuchet copient bout à bout les extraits qui les requièrent ${ }^{56}$. Dans cette configuration, Yvan Leclerc a tout à fait raison d'écrire :

Ce qui se passe dans le village ne passe plus dans le deuxième volume; ces petits événements, rumeurs lointaines, viennent mourir aux marches du Monument, en contrepoint narratif d'un morceau qui résiste à la mise en récit. Il ne s'agit plus, par attaches, liaisons, suspensions, de remédier à un défaut du sujet, d'établir un semblant de continuité en faisant appel aux personnages secondaires, Gorgu et Mélie justement, mais de montrer que le fil du collier s'est cassé. Les «attaches » qui restaient à mettre dans le second volume n'auraient pas masqué l'empilement des citations. Deux temporalités se juxtaposent sans interférences, sous le signe commun de la répétition : l'achronie de la copie, la chronique provinciale, toujours à suivre (reprise dans le « Résumé-Sommaire ", elle s'ouvre sur un « Etc. »).

S'il arrive au terme d'un long voyage (circum-navigation), le retour à la copie fait accéder à un autre ordre temporel : l'éternité : « Joie finale et éternelle ». L'une des dernières phrases de Bouvard, «Ils n'ont plus aucun intérêt dans la vie » indique $a$ contrario que l'intérêt retrouvé pour la copie ne se situe plus dans la vie, mais audelà. ${ }^{57}$

Cependant, Flaubert semble être finalement revenu sur ce dispositif et en avoir choisi un autre qui lui permet, au contraire, de ne pas extraire la copie du système fictionnel 
d'ensemble sur lequel repose le roman. Néanmoins, si la fiction était censée innerver la totalité de l'œuvre, comme on a tenté de le montrer, cela ne veut pas dire pour autant que la fiction n'aurait pas évolué voire changé de nature. En effet, dans le premier volume, la fiction se nourrit des contenus encyclopédiques patiemment amassés par Flaubert dans ses dossiers préparatoires; elle les assimile, ne laissant somme toute affleurer à la surface du texte romanesque que ce qui est utile à son propos. Cette digestion contrôlée aurait dû laisser la place, dans le second volume, à une exposition, à une ostension, voire à une projection du documentaire «brut». Il y a là un retournement, un passage de l'endroit sur l'envers, qu'ont bien perçu Claude Mouchard et Jacques Neefs :

La fiction narrative doit être la pellicule de visualisation sur laquelle l'épreuve incertaine des lectures, rassemblements, distributions de textes pourrait fugitivement se fixer: "Mais bientôt, ils éprouvent le besoin d'un classement... Mais souvent ils sont embarrassés $\mathrm{p}$. [classer] ranger le fait à sa place » (Ms gg 10, f • 32). La fiction, loin d'être ce qui aveugle le préparatoire pour s'en gonfler, devient cette doublure qui mime le travail d'où elle provient, qui le dramatise. Le recours à la fiction assure le minimum de visibilité où s'éternise l'impalpable circulation des écrits: "Finir sur la vue des deux bonshommes penchés sur leur pupitre, et copiant» (Ms. gg 10, $\mathrm{f}^{\circ}$ 67) $)^{58}$.

Pousser ainsi jusqu'à ses dernières limites la logique du système présente cependant un risque. En investiguant les conséquences esthétiques ultimes du dispositif complexe sur lequel repose le second volume in fieri, et quoiqu'ils s'en défendent ${ }^{59}$, Jacques Neefs et Claude Mouchard en viennent peu ou prou à douter de la capacité qu'aurait eue Flaubert à aller jusqu'au bout de la machinerie qu'il avait lui-même mise en branle :

[...] par ce travail sur les énoncés, sur le pouvoir même de penser et de dire, le second volume n'avale-t-il pas sa propre possibilité ?

[...] Quel volume imaginer pour contenir l'égalité généralisée, sans dehors?

Les personnages, en se chargeant d'emplir la page, auraient-ils pu assumer et dissoudre le souci du contour ${ }^{60}$ ?

Alors, la mort a-t-elle sauvé Flaubert d'un désastre ? Se serait-il trouvé dépassé par les prolongements formidables et vraisemblablement pour partie imprévus de sa propre création, tout comme son personnage Homais avait failli lui échapper dans un scénario non repris de la fin de Madame Bovary ${ }^{61}$ ? A-t-il vu trop loin? A-t-il visé trop haut? La question restera évidemment à jamais ouverte.

40 Cependant, tirer le fil d'Ariane de la fiction a permis de prendre la mesure de l'aporie à laquelle aurait pu conduire la logique sur laquelle repose le second volume, telle qu'on la découvre à l'œuvre dans les scénarios, et si on la pousse dans ses ultimes retranchements. Ce parcours présente aussi l'intérêt de rendre particulièrement sensible cette dimension de la fiction qui est à la fois l'ossature infiniment nécessaire - mais à jamais absente - du dispositif prévu par Flaubert.

On peut alors, à l'inverse, et parce que l'on est maintenant pleinement conscient du caractère exclusivement expérimental et irrémédiablement incomplet des résultats obtenus, investiguer l'autre versant du projet flaubertien, celui qui est constitué par les documents préparatoires laissés par l'écrivain. Grâce à l'outil informatique, on est aujourd'hui en situation de tester les configurations multiples qu'aurait pu prendre le second volume de Bouvard et Pécuchet en fonction de ce que contiennent les dossiers documentaires conservés à la bibliothèque de Rouen. Quand les éditions imprimées doivent choisir un agencement unique et s'y tenir, l'édition électronique offre l'opportunité de construire autant de seconds volumes différents que le lecteur a 
d'hypothèses scientifiques à tester ${ }^{62}$. Si certaines configurations seront toujours plus légitimes et pertinentes que d'autres - et si toutes sont, quoi qu'il en soit, uniformément marquées au coin de la conjecture, le dispositif lui-même, en intégrant dans sa conception les dimensions complémentaires du multiple et du mouvant, est le seul à consonner pertinemment avec le double inachèvement du roman, tant encyclopédique que fictionnel. Le dispositif éditorial se fait alors outil de composition et de structuration de l'œuvre éditée elle-même ${ }^{63}$.

\section{ANNEXES}

\section{Annexe 1}

Ms g226 (8) $\mathrm{f}^{\circ} 38-39 \mathrm{v}^{\circ}$ : inventaire établi par la nièce de Flaubert, Caroline Commanville, fin 1880-1881?

\begin{tabular}{|c|c|c|c|c|}
\hline & $\begin{array}{l}\text { Intitulés indiqués } \\
\text { par Caroline }\end{array}$ & $\begin{array}{l}\text { Volumétrie } \\
\text { indiquée par } \\
\text { Caroline }\end{array}$ & $\begin{array}{l}\text { Identification } \\
\text { typologique }\end{array}$ & Pages concernées \\
\hline \multicolumn{5}{|l|}{ g226_8_f_038__r } \\
\hline & $\begin{array}{l}\text { Bizarreries } \\
\text { Nomenclatures }\end{array}$ & $\varnothing$ & Page de titre & g226_1_f_273_rr \\
\hline & Beautés & $\varnothing$ & Page de titre & g226_1_f_166__r \\
\hline & $\begin{array}{l}\text { Histoire et idées } \\
\text { scientifiques }\end{array}$ & $\varnothing$ & Page de titre & g226_4_f_001_rr \\
\hline & $\begin{array}{l}\text { Bizarreries } \\
\text { nomenclatures }\end{array}$ & 19 feuilles & Dossier & $\begin{array}{l}\text { g226_1_f_274__r à } \\
\text { g226_1_f_292b_r }\end{array}$ \\
\hline & Beautés & $\begin{array}{l}53 \text { et plusieurs } \\
\text { feuillets dans } 1 \\
\text { enveloppe }\end{array}$ & Dossier & $\begin{array}{l}\text { g226_1_f_167__r à } \\
\text { g226_1_f_221__r }\end{array}$ \\
\hline & \multirow{2}{*}{ Histoire } & \multirow{2}{*}{25} & Page de titre & g226_4_f_016_rr \\
\hline & & & Dossier & $\begin{array}{l}\text { g226_4_f_017__r à } \\
\text { g226_4_f_040__r }\end{array}$ \\
\hline & \multirow[b]{2}{*}{ Idées scientifiques } & \multirow[b]{2}{*}{22} & Page de titre & g226_4_f_040b_r \\
\hline & & & Dossier & $\begin{array}{l}\text { g226_4_f_041_rr à } \\
\text { g226_4_f_062__v }\end{array}$ \\
\hline
\end{tabular}




\begin{tabular}{|c|c|c|c|c|}
\hline & $\begin{array}{l}\text { Bévues historiques } \\
\text { et géographiques }\end{array}$ & 5 & $\begin{array}{l}\text { mêlées avec } \\
\text { d'autres } \\
\text { choses }\end{array}$ & $\begin{array}{l}\text { g226_4_f_002_rr à } \\
\text { g226_4_f_015__r }\end{array}$ \\
\hline & & 38 feuilles & Page de titre & g226_4_ff_133__r \\
\hline & République de 1848 & $\begin{array}{l}18 \text { feuillets de } \\
\text { toutes grandeurs }\end{array}$ & Dossier & $\begin{array}{l}\text { g226_4_f_135_rr à } \\
\text { g226_4_f_204__v }\end{array}$ \\
\hline \multicolumn{5}{|l|}{ g226_8_f_038_-v } \\
\hline & $\begin{array}{l}\text { Grands hommes } \\
\text { Esthétique } \\
\text { critique } \\
\text { Styles }\end{array}$ & $\varnothing$ & Page de titre & g226_3_f_047__r \\
\hline & \multirow[b]{2}{*}{ Grands hommes } & \multirow[b]{2}{*}{30 feuilles } & Page de titre & g226_3_f_010_r \\
\hline & & & Dossier & $\begin{array}{l}\text { g226_3_f_011__r à } \\
\text { g226_3_f_046__r }\end{array}$ \\
\hline & $\begin{array}{l}\text { Esthétique et } \\
\text { Critiques }\end{array}$ & $\varnothing$ & Page de titre & g226_3_f_087__r \\
\hline & \multirow[b]{2}{*}{ Esthétique } & \multirow{2}{*}{$\begin{array}{l}15 \text { feuilles } \\
6 \text { feuillets }\end{array}$} & Page de titre & g226_3_f_048__r \\
\hline & & & Dossier & $\begin{array}{l}\text { g226_3_f_049__r à } \\
\text { g226_3_f_064__r }\end{array}$ \\
\hline & \multirow[b]{2}{*}{ Critique } & \multirow[b]{2}{*}{12 feuilles } & Page de titre & g226_3_f_065_-r \\
\hline & & & Dossier & $\begin{array}{l}\text { g226_3_f_066__r à } \\
\text { g226_3_f_086__r }\end{array}$ \\
\hline & Styles & $\varnothing$ & Page de titre & g226_3_f_117_rr \\
\hline & \multirow[b]{2}{*}{ officiel - Souverains } & \multirow[b]{2}{*}{11 feuilles } & Page de titre & g226_3_f_169_rr \\
\hline & & & Dossier & $\begin{array}{l}\text { g226_3_f_170__r à } \\
\text { g226_3_f_183__r }\end{array}$ \\
\hline & \multirow{2}{*}{ romantiques } & \multirow{2}{*}{$\begin{array}{l}5 \text { feuillets } \\
7 \text { feuilles }\end{array}$} & Page de titre & g226_3_f_150_rr \\
\hline & & & Dossier & $\begin{array}{l}\text { g226_3_f_151_rr à } \\
\text { g226_3_f_159__r }\end{array}$ \\
\hline & \multirow[b]{2}{*}{ révolutionnaires } & \multirow[b]{2}{*}{4 id [feuilles] } & Page de titre & g226_3_f_145_-r \\
\hline & & & Dossier & $\begin{array}{l}\text { g226_3_f_146__r à } \\
\text { g226_3_f_149__r }\end{array}$ \\
\hline & & & Page de titre & g226_4_f_073__r \\
\hline
\end{tabular}

Journalistes

3 [feuilles] 


\begin{tabular}{|c|c|c|c|c|}
\hline & & & Dossier & $\begin{array}{l}\text { g226_4_ffo74__r à } \\
\text { g226_4_f_076__r }\end{array}$ \\
\hline & \multirow[b]{2}{*}{ Scientifiques } & \multirow[b]{2}{*}{6 [feuilles] } & Page de titre & g226_3_f_118_r \\
\hline & & & Dossier & $\begin{array}{l}\text { g226_3_f_119_rr à } \\
\text { g226_3_f_136__r }\end{array}$ \\
\hline & \multirow[b]{2}{*}{ Ecclésiastiques } & \multirow[b]{2}{*}{6 [feuilles] } & Page de titre & g226_3_f_137_r \\
\hline & & & Dossier & $\begin{array}{l}\text { g226_3_f_138_rr à } \\
\text { g226_3_f_143__r }\end{array}$ \\
\hline & \multirow[b]{2}{*}{ Rococo } & \multirow[b]{2}{*}{14 [feuilles] } & Page de titre & g226_7_f_001_rr \\
\hline & & & Dossier & $\begin{array}{l}\text { g226_7_ffo02_rr à } \\
\text { g226_7_f_021__r }\end{array}$ \\
\hline & \multirow[b]{2}{*}{ Dramatiques } & \multirow[b]{2}{*}{6 [feuilles] } & Page de titre & g226_3_f_160_r \\
\hline & & & Dossier & $\begin{array}{l}\text { g226_3_f_161_rr à } \\
\text { g226_3_f__168__r }\end{array}$ \\
\hline & \multirow[b]{2}{*}{ Classiques corrigés } & \multirow[b]{2}{*}{5 [feuilles] } & Page de titre & g226_2_f_013__r \\
\hline & & & Dossier & $\begin{array}{l}\text { g226_2_f_014b_r à } \\
\text { g226_2_f_018__r }\end{array}$ \\
\hline & \multirow[b]{2}{*}{ grands Écrivains } & \multirow{2}{*}{$\begin{array}{l}26 \text { [feuilles] } \\
9 \text { feuillets }\end{array}$} & Page de titre & g226_3_f_088_rr \\
\hline & & & Dossier & $\begin{array}{l}\text { g226_3_f_089_rr à } \\
\text { g226_3_f_116__r }\end{array}$ \\
\hline & \multirow[b]{2}{*}{ Périphrases } & \multirow[b]{2}{*}{ 7. [feuilles] } & Page de titre & g226_3_f_001_rr \\
\hline & & & Dossier & $\begin{array}{l}\text { g226_3_f_002_rr à } \\
\text { g226_3_f_009__r }\end{array}$ \\
\hline \multicolumn{5}{|l|}{ g226_8_f_039_rr } \\
\hline & $\begin{array}{l}\text { Le Dictionnaire des } \\
\text { Idées reçues. }\end{array}$ & 40 feuilles. & $\begin{array}{l}\text { Dossier sans } \\
\text { page de titre }\end{array}$ & $\begin{array}{l}\text { g227_f_020_rr à } \\
\text { g227_f_059__r }\end{array}$ \\
\hline & \multirow{2}{*}{ Journaux - } & \multirow{2}{*}{43 feuilles } & Page de titre & g226_4_f_089__r \\
\hline & & & Dossier & $\begin{array}{l}\text { g226_4_ffo90_rr à } \\
\text { g226_4_f_132__v }\end{array}$ \\
\hline & \multirow{2}{*}{ Morale } & \multirow{2}{*}{$\begin{array}{l}14 \text { id [feuilles] } \\
3 \text { feuillets }\end{array}$} & Page de titre & g226_5_f_234__r \\
\hline & & & Dossier & $\begin{array}{l}\text { g226_5_f_235_rr à } \\
\text { g226_5_f_250__r }\end{array}$ \\
\hline
\end{tabular}




\begin{tabular}{|c|c|c|c|c|}
\hline & \multirow{2}{*}{$\begin{array}{l}\text { Religion } \\
\text { Mysticisme } \\
\text { Prophéties }\end{array}$} & \multirow{2}{*}{$\begin{array}{l}18 \text { feuilles } \\
5 \text { feuillets }\end{array}$} & Page de titre & g226_6_f_178_r \\
\hline & & & Dossier & $\begin{array}{l}\text { g226_6_f_179_r à } \\
\text { g226_6_f_197__r }\end{array}$ \\
\hline & \multirow{2}{*}{$\begin{array}{l}\text { Morale - Socialisme } \\
\text { - Politique }\end{array}$} & \multirow[b]{2}{*}{25 feuilles } & Page de titre & g226_5_f_252_r \\
\hline & & & Dossier & $\begin{array}{l}\text { g226_5_f_254_rr à } \\
\text { g226_5_f_283__r }\end{array}$ \\
\hline & $\begin{array}{l}\text { Amour - Philosophie } \\
\text { Mysticisme Religion } \\
\text { Prophéties } \\
\text { exaltation du bas. } \\
\text { Imbéciles - Esprit } \\
\text { des journaux }\end{array}$ & $\varnothing$ & Page de titre & g226_1_f_077_rr \\
\hline & \multirow[b]{2}{*}{ Amours } & \multirow[b]{2}{*}{5 feuilles } & Page de titre & g226_1_f_072_r \\
\hline & & & Dossier & $\begin{array}{l}\text { g226_1_f_073_r à } \\
\text { g226_1_f_076__r }\end{array}$ \\
\hline & \multirow[b]{2}{*}{ Philosophie } & \multirow[b]{2}{*}{4 [feuilles] } & Page de titre & g226_1_f_102_rr \\
\hline & & & Dossier & $\begin{array}{l}\text { g226_1_f_103_r à } \\
\text { g226_1_f_106__r }\end{array}$ \\
\hline & \multirow[b]{2}{*}{ Exaltation du bas } & \multirow[b]{2}{*}{8 [feuilles] } & Page de titre & g226_1_f_092_rr \\
\hline & & & Dossier & $\begin{array}{l}\text { g226_1_f_093_r à } \\
\text { g226_1_f_101__r }\end{array}$ \\
\hline & \multirow[b]{2}{*}{ Imbéciles } & \multirow[b]{2}{*}{9 [feuilles] } & Page de titre & g226_1_f_081_rr \\
\hline & & & Dossier & $\begin{array}{l}\text { g226_1_f_082_rr à } \\
\text { g226_1_f_089__r }\end{array}$ \\
\hline & \multirow[b]{2}{*}{ Esprit des Journaux } & \multirow[b]{2}{*}{4 [feuilles] } & Page de titre & g226_1_f_080b_r \\
\hline & & & Dossier & $\begin{array}{l}\text { g226_1_f_090_rr à } \\
\text { g226_1_f_091__r }\end{array}$ \\
\hline \multirow[t]{3}{*}{ g226_8_f_039__v } & & & & \\
\hline & Annexe du plan - & & Page de titre & \\
\hline & Résumé sommaire & 4 pages & $\begin{array}{l}\text { Pages } \\
\text { scénariques }\end{array}$ & $\begin{array}{l}\operatorname{gg} 10 f^{\circ} 63,64,65 \text { et } \\
66\end{array}$ \\
\hline
\end{tabular}




\begin{tabular}{|c|c|c|c|}
\hline $\begin{array}{l}\text { plan du ch. XI, } \\
\text { du ch. XII } \\
\text { qui est la } \\
\text { conclusion. }\end{array}$ & $\begin{array}{l}\text { une grande } \\
\text { [page] }\end{array}$ & $\begin{array}{l}\text { Page } \\
\text { scénarique }\end{array}$ & gg $10 \mathrm{f}^{\circ} 67$ \\
\hline $\begin{array}{l}\text { plan des morceaux } \\
\text { littéraires } \\
\text { particuliers* à } \\
\text { intercaler dans le } \\
\text { second volume }\end{array}$ & une autre [page] & $\begin{array}{l}\text { Page } \\
\text { scénarique }\end{array}$ & $\begin{array}{l}\text { g226_2_f_014_r? } \\
\text { gg10 fo } 68\end{array}$ \\
\hline $\begin{array}{l}\text { relatifs au second } \\
\text { volume }\end{array}$ & 2 feuillets & $\begin{array}{l}\text { Pages } \\
\text { scénariques }\end{array}$ & gg10 fo 46 verso? \\
\hline $\begin{array}{l}\text { de l'écriture de } \\
\text { Duplan - }\end{array}$ & $\begin{array}{l}13 \text { feuilles de } 4 \\
\text { pages presque } \\
\text { toutes relevés des } \\
\text { articles - extraits } \\
\text { de la critique sur } \\
\text { les ouvrages de } \\
\text { Flaubert }\end{array}$ & $\begin{array}{l}\text { Dossier sans } \\
\text { page de titre }\end{array}$ & $\begin{array}{l}\text { g226_8_f_208__r à } \\
\text { g226_8_f_232__r }\end{array}$ \\
\hline $\begin{array}{l}\text { L'Album de la } \\
\text { Marquise }\end{array}$ & $\varnothing$ & $\begin{array}{l}\text { Dossier sans } \\
\text { page de titre }\end{array}$ & $\begin{array}{l}\text { g226_5_f_142__r à } \\
\text { g226_5_f_147__r }\end{array}$ \\
\hline
\end{tabular}

\section{Annexe 2}

\section{Descriptif des dossiers établi par Maupassant ${ }^{64}$, publié en 1884}

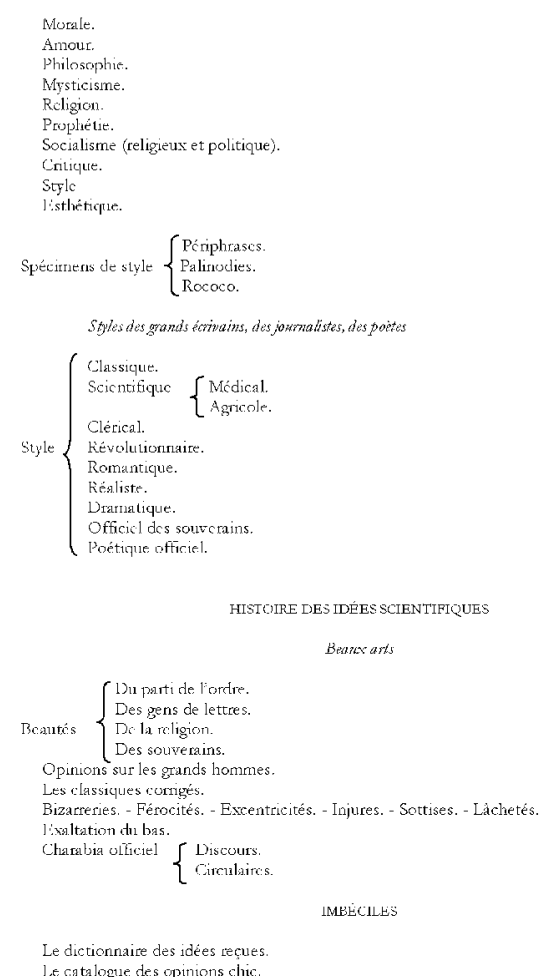




\section{NOTES}

1. Par " premier volume ", on entend les dix premiers chapitres du roman, qui ont été à peu près complètement rédigés par Flaubert avant sa mort (sauf pour la fin du dixième), et qui constituent le texte usuellement édité sous le titre de Bouvard et Pécuchet.

2. Replaçant la question du second volume sous le jour spécifique des rapports de la fiction et de l'encyclopédie, cette étude reprend et développe certains aspects de l'article de Claude Mouchard et Jacques Neefs : «Vers le second volume : Bouvard et Pécuchet ", paru dans le recueil collectif Flaubert à l'œuvre (Flammarion, «Textes et manuscrits », 1980, p. 171-217), ainsi que de l'ouvrage d'Yvan Leclerc, La Spirale et le Monument. Essai sur Bouvard et Pécuchet de Gustave Flaubert (SEDES, "Présences critiques", 1988). Cette étude reconnaît bien volontiers sa dette envers les éclairantes synthèses offertes par ces travaux, et souscrit aux aperçus toujours hautement suggestifs qu'ils prodiguent. Elle met néanmoins au service de son propos les avancées scientifiques récemment obtenues grâce à l'édition intégrale en ligne des dossiers documentaires de Bouvard et Pécuchet, projet financé par l'Agence nationale de la recherche et mené par une équipe internationale sous la direction de Stéphanie Dord-Crouslé (ouverture partielle du site au public : janvier 2012). Les premiers résultats de ce travail ont été publiés dans Éditer le chantier documentaire de Bouvard et Pécuchet. Explorations critiques et premières réalisations numériques, textes réunis par Stéphanie Dord-Crouslé, Stella Mangiapane et Rosa Maria Palermo Di Stefano, Messine, Andrea Lippolis Editore, 2010.

3. Pour l'histoire précise de l'entrée de ces manuscrits dans cette institution de conservation, on se reportera à l'article très documenté de Marie-Dominique Nobécourt-Mutarelli : «Histoire d'une transmission» (2003, en ligne sur le site Flaubert de l'université de Rouen) auquel nous empruntons notre information.

4. Pour avoir accès au descriptif complet du fonds, voir le Catalogue des manuscrits de Gustave Flaubert conservés à la Bibliothèque municipale de Rouen, rédigé par Marie-Dominique Nobécourt, mis à jour et complété le 16 mars 2005 par Claudine Brabetz, Michaël Monnier et Christelle Quillet (Bibliothèque municipale de Rouen), et Yvan Leclerc (Université de Rouen), en ligne sur le site Flaubert de l'université de Rouen.

5. Les documents conservés sous ces trois cotes sont l'objet de l'édition signalée plus haut ( http://www.dossiers-flaubert.fr/). Les liens inclus dans le présent article mènent directement aux documents mentionnés.

6. Au premier abord, le nombre de folios ne parait pas correspondre. Mais le rédacteur de l'inventaire originel semble n'avoir compté, dans ce recueil composé de feuilles de papier lignées relativement épaisses et pliées en deux comme dans un cahier d'écolier, que les folios portant du texte, tandis que lors de la foliotation patrimoniale, tous les folios ont été numérotés, y compris ceux qui sont vierges. Si l'on exclut les folios vierges, on aboutit bien à un recueil de 19 pages rédigées (soit les folios 208, 210, 212, 214, 215, 216, 217, 219, 221, 222, 223, 224, 225, 226, 227, 228, 229,231 et 232).

7. Les plans et scénarios (gg10) et les brouillons (g225) du roman font actuellement l'objet d'une édition en ligne intégrale menée sous la direction d'Yvan Leclerc (Université de Rouen).

8. Dans le tableau comparatif, les références à ces volumes sont en caractères gras. Le premier plan du roman (voir plus bas), des bribes scénariques et des citations éparses se trouvent aussi dans les carnets de travail du romancier conservés à la Bibliothèque historique de la ville de Paris (voir l'édition de Pierre-Marc de Biasi, Balland, 1989).

9. Voir l'analyse concordante de Lea Caminiti et Alberto Cento dans leur édition : Le Second volume de Bouvard et Pécuchet, le projet du Sottisier, reconstitution conjecturale de la "copie» des deux bonshommes d'après le dossier de Rouen, Naples, Liguori, 1981, p. LXXVII-XC. 
10. Ce dossier a été transcrit et analysé par Adrianne Tooke dans son ouvrage: Flaubert and the Pictorial Arts. From Image to Text, Oxford, Oxford University Press, 2000.

11. Yvan Leclerc : "La section "Théâtre" dans les dossiers de Bouvard et Pécuchet ", dans Éditer le chantier documentaire de Bouvard et Pécuchet. Explorations critiques et premières réalisations numériques, op. cit., p. 121-126.

12. Si l'« Album de la marquise» est mentionné par Flaubert dans le carnet de travail où se trouve le plan primitif de Bouvard et Pécuchet (voir plus bas), cet élément disparait ensuite des plans et scénarios ultérieurs. La dernière mention de l'album dans la correspondance de Flaubert date du 14 mars 1868. Il écrit alors à Duplan : «Le sieur Rolland [...] a remporté une veste insigne, au Vaudeville. Son œuvre fourmille de jolies phrases dont tu pourras orner l'album de la vicomtesse" (Correspondance [Corr.], édition de Jean Bruneau et Yvan Leclerc pour la «Bibliothèque de la Pléiade », Gallimard, 1973-2007, III, p. 374). Après la mort de Duplan, en 1870, il n'en est plus jamais question. Comme l'ont indiqué Lea Caminiti et Alberto Cento avec raison dans leur édition (op.cit., p. LXXXV-LXXXVI), cet élément, au moment de la mort de l'écrivain, n'était plus destiné, depuis longtemps, à entrer tel quel dans la composition du second volume. On verra néanmoins que la nièce Caroline le convoque dans son inventaire des dossiers concernés par le « volume des notes".

13. Sur ces processus concurrents et complémentaires, voir Norioki Sugaya, «Régularités et distorsions : les transferts d'extraits dans le dossier médical de Bouvard et Pécuchet ", Éditer le chantier documentaire de Bouvard et Pécuchet. Explorations critiques et premières réalisations numériques, op. cit., p. 215-228; et Stéphanie Dord-Crouslé, «Entre notes de lecture et fragments préparés pour le second volume, les transferts de citations à l'épreuve du dossier Religion » (ibid., p. 81-95).

14. Flaubert utilise cette expression pour décrire le processus rédactionnel qui est le sien dans une lettre à Louise Colet du 15 avril 1852 (« Tu verras par quelle mécanique compliquée j’arrive à faire une phrase »; Corr., II, p. 71).

15. René Descharmes et René Dumesnil, Autour de Flaubert. Études historiques et documentaires, Mercure de France, 1912, t. 2, p. 81, note 1.

16. René Dumesnil avait épousé la fille d'Edmond Laporte et a donc eu accès aux papiers de son beau-père décédé en 1906. Mais, comme le précise Jean Bruneau, cet accès n'a été que momentané puisque René Dumesnil a divorcé avant 1933 (Corr., III, p. 1410-1411, note 3).

17. Voir Stéphanie Dord-Crouslé : « Un dossier flaubertien mal connu : les notes pour le chapitre "Littérature" de Bouvard et Pécuchet "; Histoires littéraires, n² 24, 2005, p. 119-135 [disponible sur HAL-SHS].

18. À ce propos, voir Claudine Gothot-Merch, son édition du roman, Gallimard, coll. "Folio », 1979, p. 433-437.

19. Outre les pages du carnet de travail numéroté 19 et celles du recueil gg10 que nous commenterons plus bas, d'autres pages à caractère scénarique se trouvent disséminées dans les dossiers de Rouen, par exemple : volume 1 , verso du béquet du $\mathrm{f}^{\circ} 180$; volume $7, \mathrm{f}^{\circ} 321 \mathrm{r}^{\circ}$ et $\mathrm{v}^{\circ}$; et volume $8, \mathrm{f}^{\circ} 31 \mathrm{r}^{\circ}$ et $\mathrm{v}^{\circ}$.

20. Cet accord n'avait cependant pas été formalisé (voir Corr., V, p. 706.)

21. Cité par Yvan Leclerc dans son édition de la Correspondance croisée de Flaubert et Maupassant, Flammarion, 1993, p. 260.

22. L'existence de cet inventaire a été signalée pour la première fois par Claudine Gothot-Mersch dans son édition du roman (op. cit., p. 431-432).

23. Voir par exemple la dernière page du manuscrit de La Découverte de la vaccine, conservé à la BnF et en ligne sur Gallica, ou la page liminaire («Papiers trouvés ça et là sur la table de travail », $\mathrm{f}^{\circ}$ 284) d'un dossier constitué par Caroline et conservé dans le septième volume de la cote g226.

24. Voir la composition plus précise de ce document dans l'annexe 1. 
25. Actuellement, le dossier qui porte ce titre est constitué de feuillets de carnet découpés et surtout de notes de lecture destinées à L'Éducation sentimentale. On ne comprend pas pourquoi Caroline en fait état dans son inventaire.

26. Ce dossier regroupe des notes de lecture prises pour L'Éducation sentimentale et relues ensuite dans la perspective de Bouvard et Pécuchet (premier et second volume - comme le montrent les croix et les annotations portées dans les marges). Il ne se distingue pas des autres dossiers thématiques de notes de lecture (comme "Agriculture", "Médecine», etc.) si ce n'est par l'absence d'un sommaire liminaire récapitulant les titres d'ouvrages lus. Là non plus, on ne comprend pas pourquoi Caroline l'a retenu dans son inventaire.

27. Voir la note d'Yvan Leclerc dans son édition de la Correspondance croisée de Flaubert et Maupassant, op. cit., p. 260. En outre, dans son article du Gaulois paru le 6 avril 1881 (« Bouvard et Pécuchet »), Maupassant pense encore pouvoir publier le recueil de notes : "Ce dossier de la bêtise forme aujourd'hui une montagne de notes. Peut-être, l'an prochain, pourra-t-il être livré au public » (ibid., p. 285). Lorsqu'il reprend la formule en 1884 dans la Revue bleue, elle est devenue : «Ce dossier de la bêtise humaine formait une montagne de notes demeurées trop éparses, trop mêlées, pour être jamais publiées en entier » (ibid., p. 299).

28. «Si vous [...] aviez besoin tout de suite [de ces manuscrits], voulez-vous les faire prendre chez moi ? Ils sont sur un petit meuble dans mon cabinet de travail. Ma concierge les remettra " (lettre de Maupassant à Caroline du 30 juillet 1881 ; ibid., p. 261-262). Les citations suivantes sont extraites de la même lettre.

29. En 1884, cette étude a été prépubliée avec des coupes dans la Revue bleue puis a paru in extenso en préface aux Lettres de Gustave Flaubert à George Sand, chez Charpentier.

30. On le trouvera reproduit dans l'annexe 2.

31. Pour être complet, il faudrait évoquer les descriptifs des dossiers qu'ont successivement donnés l'éditeur Conard, dans la notice publiée à la suite du roman (1910, p. 402-408) ; E.-L. Ferrère dans sa thèse sur le Dictionnaire des idées reçues (1913); René Descharmes et René Dumesnil dans leurs divers ouvrages et édition (Belles-Lettres, 1945); et Louis Demorest dans À travers les plans manuscrits et dossiers de Bouvard et Pécuchet (Les presses modernes, 1931).

32. Correspondance croisée de Flaubert et Maupassant, op. cit., p. 260.

33. Ibid., p. 308.

34. Les notes de lecture prises sur cet ouvrage (volume 7 , fo $184-185$ ) permettent de localiser précisément l'extrait que le romancier n'a pas recopié in extenso mais qu'il commente avec vivacité : « histoire d'amour de l'invention du sieur Proudhon - conte à la La Bruyère ! - morceau qu'il faut lire pr connaître ce que c'est que le style des penseurs ! "La blonde Marie était aimée du jeune Maxime - Marie simple ouvrière - Maxime laborieux artisan unissant la raison à la jeunesse". - etc. - p. $53 »\left(f^{\circ} 184 v^{\circ}\right)$.

Voici le texte visé par Flaubert :

«La blonde Marie était aimée du jeune Maxime ; Marie, simple ouvrière, et dans la naïveté d'un premier amour ; Maxime, laborieux artisan, unissant la raison à la jeunesse. La nature semblait avoir prédestiné ces amants au bonheur, en les douant tous deux de simplicité et de modestie. Assidus au travail tous les jours de la semaine, Maxime s'efforçait d'augmenter son épargne ; Marie tressait en silence sa couronne de mariage. Ils ne se voyaient que le dimanche ; mais qu'il était beau, qu'il était solennel pour eux, ce jour où il fut chanté dans le ciel : L'amour est plus fort que la mort! qu'il répandait sur leur tendresse mutuelle de religion et d'innocence! Amants véritables ne furent jamais sacrilèges: pleins d'un amoureux respect, qu'aurait osé le jeune homme? qu'aurait permis la jeune fille belle de sa pudeur et de la joie du dimanche? Seuls avec leur amour, ils étaient sous la garde de Dieu. La révolution de juillet vint brusquement détruire tant de félicité. Maxime fut averti de se pourvoir: plus d'ouvrage, plus de joie. Il résolut de s'éloigner pour un temps et de se diriger vers la capitale. La veille de son départ, un dimanche au soir, il saisit la main de Marie, et, sans lui parler, la conduisit à l'église. - «Si je reviens fidèle, 
quelle vous retrouverai-je, Marie? [- ] Faites ce que vous dites, et comptez sur ma foi. - «le promettez-vous devant Dieu?» Elle le promit. Ils sortirent ; la nuit était belle ; Maxime, selon la coutume des amants qui se séparent, fit voir à Marie l'étoile polaire et lui apprit à en reconnaitre la position. - [«] Vos yeux ne rencontreront plus les miens, lui dit-il, tous les dimanches, à pareille heure, je porterai mes regards de ce côté-là. Faites-en de même, afin qu'au même instant, comme nos cœurs sont unis, nos pensées se confondent. C'est tout ce que je demande, jusqu'à ce que je vous revoie. » Il partit. Paris ne lui donna pas toujours de l'ouvrage ; ses jours de chômage lui devinrent funestes. Par les instigations de quelques amis, Maxime fut affilié à une société républicaine... Une invincible mélancolie s'empara de son âme et altéra son caractère. "Savezvous, écrivait-il à Marie, pourquoi vous êtes si pauvre, lorsque tant d'effrontées vivent dans le luxe? pourquoi je ne puis vous épouser, lorsque tant d'hommes se précipitent dans le libertinage ?... Savez-vous pourquoi je travaille quelquefois le dimanche, tandis que d'autres jouissent ou s'ennuient toute la semaine ?... Dieu a permis que les bons fussent les premiers à pâtir des vices des méchants, pour leur apprendre que c'est à eux d'émonder la société et de faire refleurir la vertu. Si le juste n'avait jamais à se plaindre, le pervers ne se corrigerait pas; et la contagion s'étendant toujours, le monde, bientôt tout infecté, périrait... Priez Dieu pour moi, Marie, c'est tout ce que peut une faible femme. Mais il y a un million de jeunes hommes vertueux et forts, tous prêts à se lever, et qui ont juré de sauver la nation... Nous vaincrons ou nous saurons mourir." Maxime fut tué derrière une barricade dans les journées de juin. Depuis ce temps, son amante a pris le deuil : orpheline dès son bas âge et n'ayant plus de mère, elle s'est attachée à la vieille mère de son fiancé. Ses journées se passent dans le travail et dans les soins d'un tendre dévouement. Tous les dimanches on la voit, dans l'obscure chapelle où elle promit à Maxime son cœur et sa foi, assister à l'office divin : c'est là que son âme, calme et résignée, se fortifie et s'épure dans un ineffable amour. Et le soir, après sa prière, le cœur plein des dernières paroles de Maxime, jusqu'à ce que je vous revoie, la triste Marie regarde en soupirant l'étoile polaire. » (De la célébration du dimanche, Garnier frères, 1850, p. 53-54).

35. Ces deux extraits sont mentionnés dans une lettre que Flaubert adresse à George Sand le 5 juillet 1868 (Corr., III, p. 771) ; l'écrivain y précise que le «Voyage» de Veuillot se trouve dans Ça et là. Effectivement, le livre XIV de l'ouvrage, intitulé « Contes et paysages bretons », est composé de plusieurs historiettes édifiantes dont il est impossible de dire, en l'absence des notes sûrement prises par Flaubert, lesquelles étaient plus particulièrement visées (Ça et là, $4^{\mathrm{e}}$ éd., Gaume frères et J. Duprey, 1860, t. 2, p. 329-383).

36. Lettre adressée à Edma Roger des Genettes, en date du 19 août 1872 (Corr., IV, p. 559). Dans les éditions précédant celle de la Bibliothèque de la Pléiade, il n'y avait pas de virgule après le verbe « copient ». Cette omission modifie considérablement l'interprétation du dispositif évoqué par Flaubert. Comme l'indique Yvan Leclerc, «si le second membre de phrase désigne le projet dans sa totalité, alors il faut étendre l'empire de la copie à tout ce qui s'écrit sous le titre de Bouvard I et II, et faire des deux bonshommes aussi, pourquoi pas, les copistes de cette espèce d'encyclopédie critique en farce qu'est leur propre histoire, donc les copistes du roman. Il n'y a pas une histoire romanesque suivie d'une copie de l'encyclopédie mais l'invention de l'Encyclopédie romancée ou du roman de l'Encyclopédie au sens, peut-être, où les bonshommes concluent que "la physiologie est (suivant un vieux mot) le roman de la médecine" " (La spirale et le monument..., op. cit., p. 65).

37. Voir deux lettres à Edma Roger des Genettes, l'une du $1^{\mathrm{er}}$ mai 1874 : « Dans une quinzaine je m'en retourne vers ma cabane, où je vais me mettre à écrire mes Deux Copistes » (Corr., IV, p. 793) ; et l'autre du 14 juillet 1874 : « Tout cela va me déranger de mon roman des Deux Copistes, auquel je voudrais me mettre tout de suite en arrivant à Croisset » (ibid., p. 835-836).

38. Voir le classement des scénarios par Alberto Cento dans l'introduction à son édition du roman: Bouvard et Pécuchet, édition critique précédée des scénarios inédits, Naples, Istituto 
universitario orientale, et Paris, Nizet, 1964. La transcription de ces pages est désormais aisément consultable sur le site du Centre Flaubert.

39. Lettre à Edma Roger des Genettes du 15 avril 1875 (Corr., IV, p. 920).

40. Lettre à la même du 10 novembre 1877 (Corr., V, p. 322).

41. Lettre à Caroline du 5 octobre 1872 (Corr., IV, p. 586).

42. Lettre à Edma Roger des Genettes du 15 février 1877 (Corr., V, p. 186).

43. Lettre de Tourgueneff à Flaubert du 12 juillet 1874 (Corr., IV, p. 833). Taine lui aussi défendait le parti de la brièveté : "Mon impression est que le livre, même exécuté aussi bien que possible, ne peut être bon ; le comique [que Flaubert] croit y mettre avortera forcément et, comme une fusée qui rate, fera long feu. Les deux héros étant bornés, bêtes, des personnages à la façon d'Henri Monnier, leurs déceptions et mésaventures sont nécessairement plates ; on s'y attend, elles n'intéressent pas; on voit deux escargots qui s'efforcent de grimper au sommet du Mont Blanc ; à la première chute, on sourit ; la dixième est insupportable. Un sujet pareil ne peut fournir qu'une nouvelle de cent pages au plus » (lettre à Tourgueneff, non datée, citée dans Claude Digeon, Le dernier visage de Flaubert, Aubier, 1946, p. 66-67).

44. Lettre à Tourgueneff du 29 juillet 1874 (Corr., IV, p. 843).

45. Lettre au même du 3 juillet 1875 (ibid., p. 929).

46. Lettre au même du 22 janvier 1879 (Corr., V, p. 508). Voir aussi la lettre à Edma Roger des Genettes du [9-VII-1878] : «C'est de la conception même du livre que je doute ? Il n'est plus temps d'y réfléchir. Tant pis!» (ibid., p. 402).

47. Lettre à Adèle Perrot du 17 octobre 1872 (Corr., IV, p. 589-590).

48. Lettre à Émile Zola du 5 octobre 1877 (Corr., V, p. 307).

49. Lettre à Léonie Brainne du 30 ou 31 décembre 1878 (ibid., p. 482).

50. Lettre à Edma Roger des Genettes du 18 avril 1880 (ibid., p. 886).

51. Lettre à Gertrude Tennant du 6 décembre 1879 (ibid., p. 767). Aussi Flaubert écrit-il à Zola lors de la parution d'Une page d'amour: «Plusieurs fois en vous lisant, je me suis arrêté pour vous envier et faire un triste retour sur mon roman, à moi - mon pédantesque roman ! qui n'amusera pas comme le vôtre !» (lettre écrite vers le 25 avril 1878 ; ibid., p. 378-379).

52. Un autre feuillet du carnet 19 comporte une information intéressante pour notre propos. Il s'agit du folio 29, intitulé "Pr les Deux Cloportes » et datant vraisemblablement de la même période. Il précise qu'il faudra «insérer dans leur copie: Le Dictionnaire des Idées Reçues // l'Album de la Marquise». Sur le folio 19 du recueil des plans et scénarios (gg10), Flaubert ne mentionne plus l'Album, mais toujours le Dictionnaire, cette fois-ci complété par le « catalogue des opinions chic ».

53. Ces scénarios ont été initialement transcrits et classés par Alberto Cento en préambule à son édition du roman (op. cit.).

54. «Aujourd'hui, je me suis promené dans le jardin, par un temps splendide et triste. Et j'ai lu de la philosophie médicale. - Car je commence mes grandes lectures pour Bouvard et Pécuchet. Je t'avouerai que le plan, que j'ai relu hier soir après mon dîner, m'a semblé superbe, mais c'est une entreprise écrasante et espovantable. Tu n'as pas dû y comprendre grand-chose, d'après ce que je t'en ai dit. - Et après avoir relu mes quatre pages de scénario, j'ai eu le regret de t'en avoir parlé » (lettre du 22 août 1872 ; Corr., IV, p. 561).

55. Il semble en effet qu'on puisse lui rattacher cette affirmation de l'écrivain dans une lettre à Maupassant du 15 janvier 1879] : «La fin de mon roman dépassera, comme violence, le fameux article de Zola » (Corr., V, p. 500). L'écrivain fait ici allusion à un article de Zola paru dans Le Figaro du 22 décembre 1878, "Les romanciers contemporains ", dans lequel le romancier attaque violemment les «faiseurs de romans" (voir la note d'Yvan Leclerc dans la Correspondance Flaubert-Maupassant, Flammarion, 1993, p. 410).

56. Cette difficulté est à l'origine des deux conceptions qui s'opposent sur la localisation exacte de la copie dans le plan du second volume: « on dispute encore pour savoir si elle aurait pris 
place après le chapitre XII, le chapitre XI ne comportant que des fragments (hypothèse de D.-L. Demorest à laquelle se rallie $\mathrm{Cl}$. Gothot-Mersch), ou si elle aurait constitué un monstrueux chapitre XI, le XII ${ }^{\mathrm{e}}$ apportant une rapide chute narrative (solution de Cento, qui semble confirmée par une lettre de Flaubert : "Restera le second volume, rien que des notes... Elles sont presque toutes prises. Enfin le chapitre XII sera la conclusion en trois ou quatre pages") » (Yvan Leclerc, La Spirale et le Monument, op. cit., p. 136).

57. Ibid., p. 134-135.

58. «Vers le second volume », op. cit., p. 180-181.

59. «On ne dira pas que le second volume n'aurait, de toute façon, jamais pu exister (pas plus qu'on n'a voulu décrire ce qu'il aurait été si Flaubert l'avait achevé) »(ibid., p. 188).

60. Ibid., p. 202.

61. « Homais s'absorbait dans le soleil d'Austerlitz. Doute de lui. regarde les bocaux. doute de son existence - ne suis-je qu'un personnage de roman, le fruit d'une imagination en délire, l'invention d'un petit paltaquot [paltoquet] que j'ai vu naître \& qui m'a inventé pr faire croire que je n'existe pas [?]» (gg9 fo 39, en ligne sur le site Les manuscrits de Madame Bovary, éd. Yvan Leclerc et Danielle Girard).

62. C'est le dispositif que le site d'édition des dossiers documentaires de Bouvard et Pécuchet ambitionne de mettre à la disposition de tous les visiteurs.

63. Voir Stéphanie Dord-Crouslé et Emmanuelle Morlock-Gerstenkorn: "L'image du kaléidoscope illustre bien le processus. Dispositif optique constitué d'un nombre fini d'éléments contenus dans un espace lui-même fini, mais autorisant un nombre indéfini de combinaisons entre ses éléments, le kaléidoscope présente la particularité de créer du nouveau par simple réagencement de ce qui existait déjà auparavant. Un nombre fini d'éléments produit ainsi un nombre indéfini de figures différentes. Ce ne sont donc pas les éléments qui font le tout, mais la forme que prend leur combinaison. Comme le tube du kaléidoscope, l'outil électronique de lecture permet de faire l'expérience que le tout est plus que la somme des parties. L'édition électronique d'un corpus comme celui des dossiers documentaires de Bouvard et Pécuchet ne peut se contenter d'être un outil de lecture et un outil de diffusion, elle doit également se faire outil de composition et de structuration de l'œuvre éditée elle-même » ("Sur le modèle du kaléidoscope : concevoir l'édition électronique du "second volume" de Bouvard et Pécuchet ", Nouveaux Cahiers François Mauriac, n 19 - «L'édition critique, de l'imprimé au numérique : François Mauriac et les autres... ", 2011, p. 181-182).

64. Pour l'établissement du texte, nous suivons Yvan Leclerc dans son édition de la Correspondance croisée de Flaubert et Maupassant, qui reproduit scrupuleusement l'édition originale (op. cit., p. 299-300).

INDEX

Mots-clés : fiction, scénario, génétique textuelle, manuscrit

\section{AUTEUR}

STÉPHANIE DORD-CROUSLÉ 